\title{
Approximating the Lateral Distribution Function of Cherenkov Radiation as a Function of the Particle Type for Tunka-133 Array
}

\author{
Zena Fadhel Khadhum ${ }^{1}$, Hassan Abdullah Mahdi ${ }^{1}$ and A. A. Al-Rubaiee ${ }^{2}$ \\ ${ }^{1}$ Department of Physics, College of Science, Al-Mustansiriyah University. \\ ${ }^{2}$ Department of astronomy and space, College of science, Baghdad University, Baghdad-Iraq.
}

\begin{abstract}
The main interest of the present work is in analysing the lateral distribution function (LDF) of Cherenkov radiation from particles that produced in Extensive Air Showers (EAS). The simulation of LDF of Cherenkov radiation is fullfiled by utilizing the CORSIKA programe at $3 \cdot 10^{15} \mathrm{eV}$ of the primary energy around the knee region for many primaries for vertical showers for Tunka-133 array conditions. Depending on the numerical simulation results of Cherenkov light LDF, sets of parameterized polynomial functions are resetted for several particles as a function of primary particle type. The comparison between the approximated LDF of Cherenkov radiotion with the LDF which has been simulated using CORSIKA program for Tunka-133 array is verified for several primary particles for vertical EAS cascade. [DOI: 10.22401/JNUS.21.2.14]
\end{abstract}

Keywords: Cherenkov light, lateral distribution function, Extensive Air Showers.

\section{Introduction}

Primary cosmic rays (PCRs) of energy spectrum and mass composition in EAS have been studied around the knee region. This approach has a main significant for getting information about PCR acceleration mechanisms and origin $[1,2]$. Generally, LDF of Cherenkov radiation depends on the type and energy of the produced primary particle, observation level, distance from EAS core $R$, height of the first interaction and the direction of shower axis [3,4]. Various studies on Cherenkov radiation in EAS have been performed by many authors. V. Prosin et al. have presented the results of studying CRs energy spectrum and mass composition over 3 seasons with Tunka-133 EAS array [5]. Furthermore, they have improved a method of EAS parameter reconstruction. G. Rastegarzadeh et al. have compared the Cherenkov light LDF results simulation for gamma and hadron showers with the experimented results of Tunka-like array in the energy range $100 \mathrm{GeV}-25 \mathrm{TeV}$ [6]. On the other side, I. De Mitri has studied CRs energy spectrum using ARGO-YBJ experiment in the energy range $(\mathrm{TeVs} \rightarrow \mathrm{PeVs})$ [7].

In this paper, the LDF simulation of Cherenkov radiation was fulfilled utilizing CORSIKA code around the knee region for configurations of Tunka-133 array $[8,9]$ at the energy $3 \mathrm{PeV}$ for vertical showers and several primary particles, such as: $(\mathrm{H}, \mathrm{He}, \mathrm{Li}, \mathrm{Be}, \mathrm{Ne}$, $\mathrm{Na}, \mathrm{Mg}, \mathrm{Al}, \mathrm{Sc}, \mathrm{Ti}, \mathrm{V}$ and $\mathrm{Cr}$ ). An approximating of Cherenkov light LDF has been obtained by depending on Breit-Wigner model [10, 11], by basing on numerical simulation method as a function of the particle's type.

\section{Lateral Profile of Cherenkov Light}

Cherenkov radiation can be emitted by ultrahigh energy particles (such as electrons, positrons and muons) of developing EAS, which gives information about the particle producing the cascade. Charged particle threshold energy, that excite the Cherenkov light in the atmosphere might be specified by the condition $\gamma=\gamma_{t h}$ with $\gamma=E / m c^{2}$, where $\gamma$ is the particles Lorenz factor in the laboratory system. At the threshold energy, Lorenz factor $\left(\gamma_{t h}\right)$ can be given as [12]:

$$
\gamma_{\mathrm{th}}=\frac{1}{\sqrt{1-\beta^{2}}}=\frac{1}{\sqrt{1-(1 / \mathrm{n}(\mathrm{h}))^{2}}}
$$

The condition $\gamma>\gamma_{\text {th }}$ is equal to the condition $v>c / n, \quad$ i.e. $n \beta>1$, where $\beta=\mathrm{v} / \mathrm{c}$, that means, the charged particle speed must exceed the speed of light $c$ with $n$ refractive index that depends on the height of shower $(h)[12]$ :

$$
n(h)=1+\zeta(h),
$$


Where,

$$
\zeta(h)=\zeta_{0} \exp \left(-\frac{h}{h_{0}}\right)
$$

When $\zeta_{0} \approx 3 \cdot 10^{-4}, \quad h_{0}=7.5 \mathrm{~km}$. The energy of electron in atmosphere is given as [13]:

$$
E_{t h}=m c^{2} \gamma_{t h}
$$

By substituting equation (1) in equation (3) one can get:

$$
E_{t h}=\frac{m c^{2}}{\sqrt{1-(1 / n(h))^{2}}}=m c^{2} \frac{n(h)}{\sqrt{n(h)^{2}-1}} \ldots \ldots
$$

The threshold energy of electron, which depends on the height $(h)$ in atmosphere, can be given through eq. (2) and eq. (3) where:

$$
E_{t h}(h)=m c^{2} \frac{1+\zeta}{\sqrt{2 \zeta(1+\zeta / 2)}} \approx \frac{m c^{2}}{\sqrt{2 \zeta_{0}}} \exp \left(h / 2 h_{0}\right)
$$

The Cherenkov light wil emit an electrons with energies greater than $E_{t h}$. Thus at sea level $n=1+\zeta_{o}$ and $\gamma>\gamma_{t h}=\frac{E_{t h}}{m c^{2}} \approx 40.8$ [12].

For Cherenkov photons radiation by electrons, $E_{t h}$ in the atmosphere at the highest $h$ approximately canbe defined from equation (5) at the height $h_{o}=7.5 \mathrm{~km}$.

Cherenkov radiation that initiated by particles with very high energies $\beta \approx 1$ slants under a small incident angle $\theta_{r}$, that is given by the relation [12]:

$$
\cos \theta_{r}=\frac{1}{\beta n} \approx 1
$$

From eq. (1) to eq. (6), one can find:

$$
\sin _{\theta_{r}}^{2}=2 \zeta_{0} \exp \left(-\frac{h}{h_{0}}\right)\left[1-\frac{E_{t h}^{2}(h)}{E^{2}}\right], \ldots .
$$

At sea level, when $E>>E_{t h}(h)$, one can find:

$\sin \theta \approx \theta_{\mathrm{r}} \approx \frac{1}{\gamma_{\mathrm{th}}} \approx \sqrt{2 \zeta_{0}} \exp \left(-\frac{\mathrm{h}}{2 \mathrm{~h}_{0}}\right)=2.45 \cdot 10^{-2}$

At the wavelength interval $\left(\lambda_{1}, \lambda_{2}\right)$, the Cherenkov photons quantity can be found through the Tamm-Cherenkov relation [14], where:

$$
\begin{aligned}
& \frac{d N_{\gamma}}{d x}=2 \pi \alpha \sin _{\theta_{r}}^{2} \int_{\lambda_{1}}^{\lambda_{2}} \frac{d \lambda}{\lambda^{2}}=2 \pi \alpha\left(\frac{1}{\lambda_{1}}-\frac{1}{\lambda_{2}}\right) \zeta_{0}(1- \\
& \left.\frac{E_{t h}^{2}(h)}{E^{2}}\right) \exp \left(-\frac{h}{h_{0}}\right)
\end{aligned}
$$

Where $\alpha=1 / 137$. The rate of the Cherenkov photon is:

$\frac{d N_{\gamma}}{d x}=\frac{d N_{\gamma}}{d x} \frac{d x}{d t}=x_{0} \exp \left(\frac{h}{h_{0}}\right) \frac{d N_{\gamma}}{d x}$,

Where

$\frac{d x}{d t}=x_{0} \exp \left(\frac{h}{h_{0}}\right)$

Where $x_{0}=\frac{t_{0}}{\rho_{0}} \approx 3.09 \cdot 10^{4}$, that is defined as the electrons distance at sea level; $t_{0}=37.1$ and $\rho_{0}=1.2 \cdot 10^{-3}[14]$.

The total number of photons $N_{\gamma}$ that radiated by electrons with neglecting the absorption process in atmosphere, can be defined as:

$N_{\gamma}=45 \cdot 10^{10} \frac{E_{0}}{10^{15} \mathrm{eV}}$

The LDF is a function that describes the lateral variation of Cherenkov flux with a distance from the shower axis, which is used extensively in event reconstruction, for getting an information about primary particle. Estimating of the age parameter and the core position are made also through utilizing the total number of photons in the cascade shower that is given as: $\left(E_{0}\right)$ :

$N_{\gamma}=3.7 .10^{3} \frac{E_{o}}{\beta_{t}}$,

where $\beta_{t}=\beta_{\text {ion }} t_{o}$, which it is the critical energy. For electron, $\beta_{\text {ion }}=$ $2.2 \mathrm{Mev} .\left(\mathrm{g} \cdot \mathrm{cm}^{-2}\right)^{-1}, t_{o}=37 \mathrm{~g} \cdot \mathrm{cm}^{-2}$ and $\beta_{t}=81.4 \mathrm{MeV}$ [14].

In general case, the LDF of Cherenkov radiation is depended on the primary particle energy that produced in EAS. The photons number per detector area unit is given by [12]:

$Q(E, R)=\frac{\Delta N_{\gamma}(E, R)}{\Delta S}$

where $\Delta N_{\gamma}$ is number of photons, $\Delta S$ is the area of detector. 


\section{Results and Discussion}

\subsection{Modelling and Approximating of Cherenkov Light LDF}

The LDF of Cherenkov light simulation is fulfilled using a detailed Monte Carlo program that is called CORSIKA code (Cosmic Ray Simulation for KAscade) [15], which studying the development and characteristics of EAS produced by energies up to $10^{20} \mathrm{eV}$. This simulation was fulfilled for conditions of Tunka-133 array around the knee energy region $(3 \mathrm{PeV})$ for several primary particles. Two hadronic models were used through the simulation; QGSJET code [16] that was used for modeling the hadrons interactions with high energies and GHEISHA code [17] that was used for modeling the hadrons interactions for lower energies. For approximating the simulated LDF of Cherenkov radiation. The proposed four parameters function are used and given by $[18,19]$ :

$$
Q(\eta, R)=\frac{\operatorname{C\sigma exp}[\alpha-\xi]}{\beta\left[(R / \beta)^{2}+(R-k)^{2} / \beta^{2}+R \sigma^{2} / \beta\right]}
$$

Where $\xi$ is a parameter given by:

$$
\xi=R / \beta+(R-k) / \beta+(R / \beta)^{2}+(R-k)^{2} / \beta^{2}
$$

where $C$ is the normalization constant [7]; $R$ is the distance from the shower axis; $\alpha, \beta, \sigma$ and $k$ are parameters of Cherenkov light LDF, which are approximated as a function of the primary particle, which is given by the relation:

$$
\begin{aligned}
& Y(\eta)=c_{o}+c_{1} \log _{10}(\eta)+c_{2}\left(\log _{10}(\eta)\right)^{2}+ \\
& c_{3}\left(\log _{10}(\eta)\right)^{3}
\end{aligned}
$$

where $Y(\eta)$ is presented for the parameters:

Table (1)

Coefficients $c_{i}$ which determine $\eta$ and depending on $\alpha, \beta, \sigma$ and $k$ parameters for vertical shower.

\begin{tabular}{|c|c|c|c||c||c||}
\hline \multicolumn{6}{|c|}{$\boldsymbol{\theta}^{\mathbf{9}} \mathbf{0}^{\mathbf{0}}$} \\
\hline $\mathbf{Y}$ & $\boldsymbol{c}_{\mathbf{0}}$ & $\boldsymbol{c}_{\mathbf{1}}$ & $\boldsymbol{c}_{\mathbf{2}}$ & $\boldsymbol{c}_{\mathbf{3}}$ & $\mathbf{x}^{\mathbf{2}}$ \\
\hline $\boldsymbol{\alpha}$ & $78.654 .10^{-1}$ & $19.775 .10^{-1}$ & $-73.510 .10^{-2}$ & $83.960 .10^{-3}$ & $37.3 .10^{-4}$ \\
\hline $\boldsymbol{\beta}$ & $-49.106 .10^{-2}$ & $11.263 .10^{-1}$ & $-45.005 .10^{-2}$ & $55.050 .10^{-3}$ & $34.5 .10^{-4}$ \\
\hline $\boldsymbol{\sigma}$ & $29.746 .10^{-1}$ & $-45.592 .10^{-1}$ & $18.961 .10^{-1}$ & $-24.605 .10^{-2}$ & $23.6 .10^{-4}$ \\
\hline $\boldsymbol{k}$ & $-18.761 .10-1$ & $-12.180 .10^{-3}$ & $28.143 .10^{-2}$ & $-58.120 .10^{-3}$ & $57.0 .10^{-5}$ \\
\hline
\end{tabular}

$\alpha, \log \beta, \log \sigma$ and $\log k$ (see Table 1$)$. Whereas, $c_{0}, c_{1}, c_{2}$, and $c_{3}$ are coefficients depended on the primary particle's type and presented in see Table (1).

The approximated Chrenkov light LDF in Figures 4, 5 and 6 for vertical cascades is slightly differs from that simulated for conditions of Tunka-133 array. The difference between the calculated and simulated LDF of Cherenkov radiation is specified by minimization of the formula:

$\Delta=\sum_{i}\left[\frac{Q_{p a r}(\eta, R)}{Q_{C O R}(R)}-1\right]^{2} \rightarrow \min$,

Where, $Q_{\text {par }}$ is the parameterized Cherenkov light LDF by eq. (15) and is the simulated Cherenkov light LDF by CORSIKA code. In Table (2) was demonstrated the results of minimization for each primary particle by using eq. (18).

Figures (4-6) demonstrate the parameterization of Cherenkov light LDF using Eqs. (15)-(17) in comparison with that simulated with CORSIKA programe for vertical showers and several primary particles at the energy $3 \mathrm{PeV}$. 

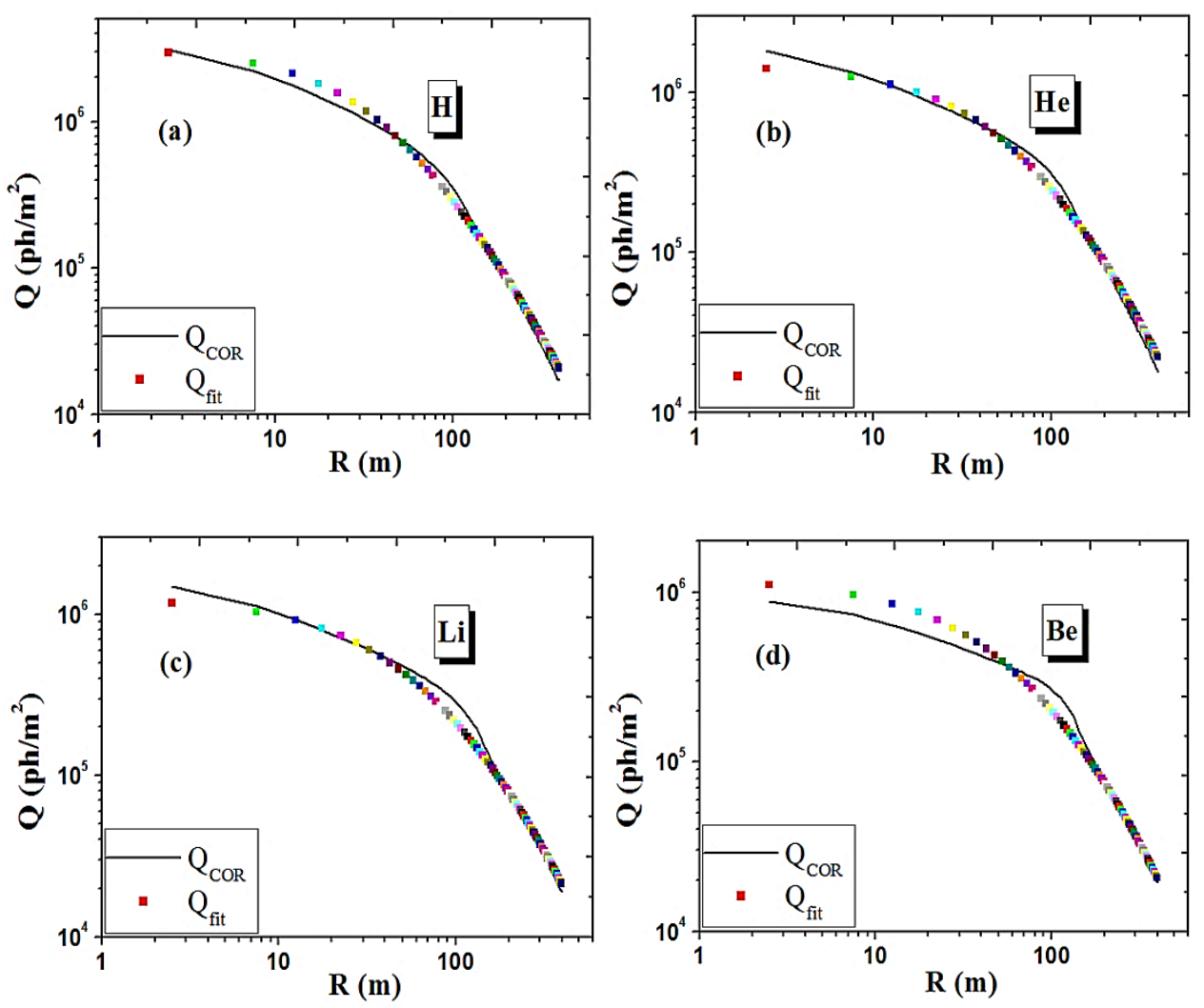

Fig.(4): Cherenkov light LDF simulation by CORSIKA program at $3 \mathrm{PeV}$ of primary energy with $\theta=0^{\circ}$ (solid curves) in comaparision with that parameterized using Eq. (15) (symbols) for: (a) H;

(b) He; (c) Li and (d) Be. Where $R(m)$ is the distance from the showers axis in meter units.
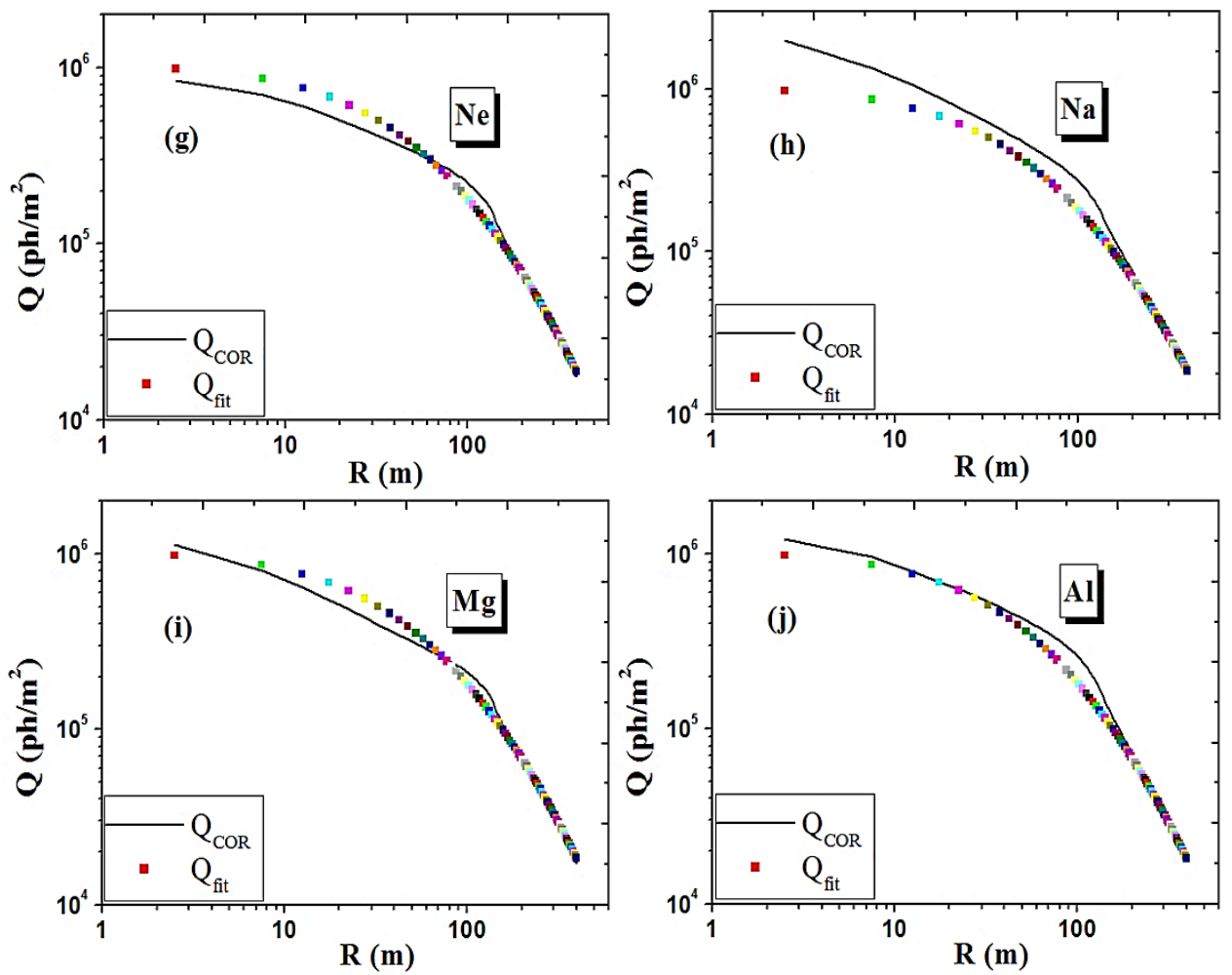

Fig.(5): Cherenkov light LDF simulation by CORSIKA program at $3 \mathrm{PeV}$ of primary energy with $\theta=0^{\circ}$ (solid curves) in comaparision with that parameterized using Eq. (15) (symbols) for: (g) Ne;

(h) Na; (i) Mg and (j) Al. Where $R(m)$ is the distance from the showers axis in meter units. 

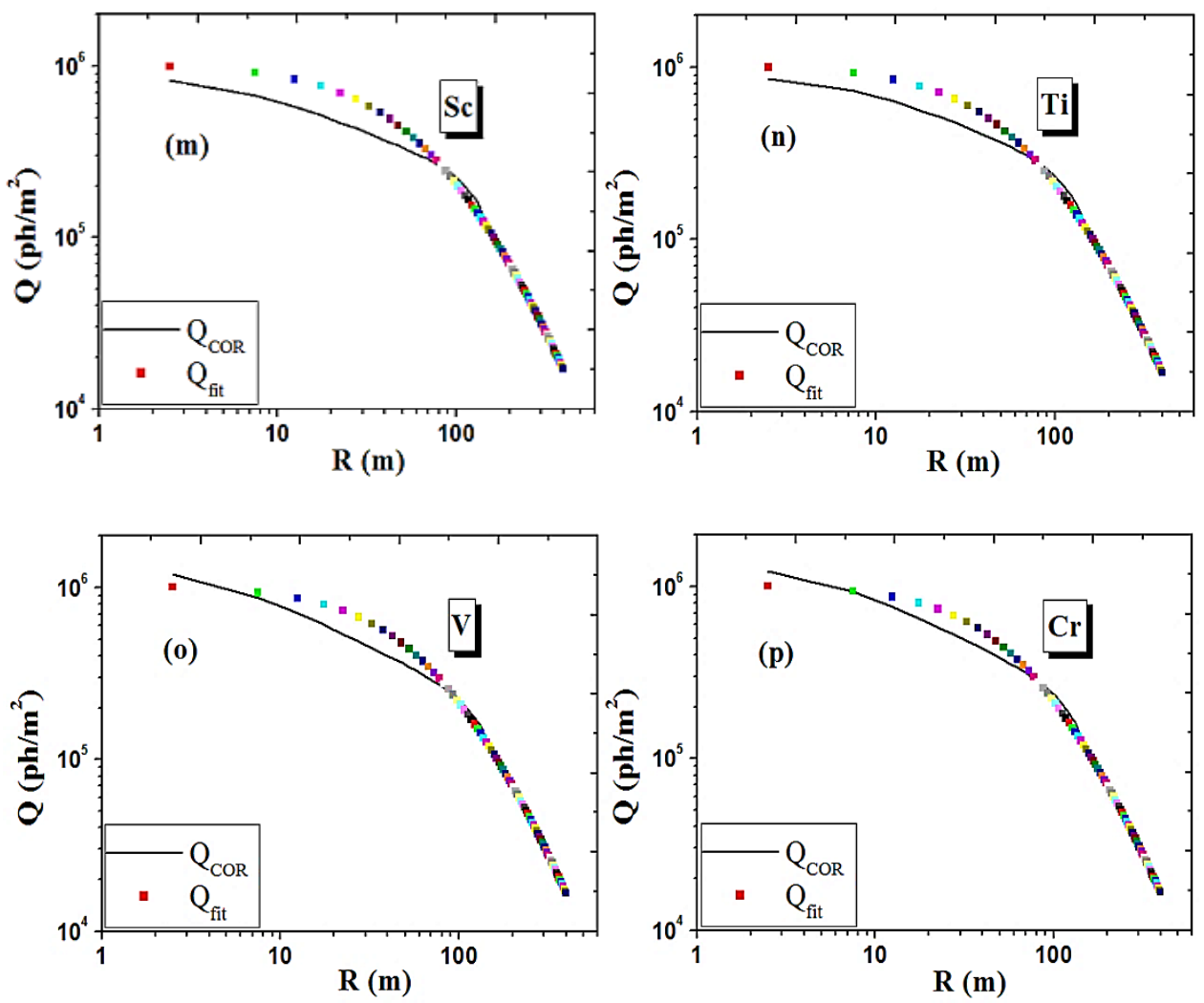

Fig.(6): Cherenkov light LDF simulation by CORSIKA program at $3 \mathrm{PeV}$ of primary energy with $\theta=0^{\circ}$ (solid curves) in comaparision with that parameterized using Eq. (15) (symbols) for: (m) Sc;

(n) Ti; (o) V and (p) Cr. Where $R(m)$ is the distance from the showers axis in meter units.

Table (2)

$\Delta_{\min }$ results that estimated with Eq. (18) for different particles at $3 \mathrm{PeV}$ primary energy.

\begin{tabular}{|l||c||c||c||}
\hline \multicolumn{1}{|c|}{ Element } & Symbol & $\mathbf{H}$ & $\Delta \mathbf{m i n}$ \\
\hline \hline Hydrogen & $\mathrm{H}$ & 101 & $14.699 .10^{-4}$ \\
\hline \hline Helium & $\mathrm{He}$ & 402 & $22.873 .10^{-4}$ \\
\hline Lithium & $\mathrm{Li}$ & 703 & $85.901 .10^{-4}$ \\
\hline Beryllium & $\mathrm{Be}$ & 905 & $12.755 .10^{-4}$ \\
\hline Neon & $\mathrm{Ne}$ & 2010 & $22.071 .10^{-4}$ \\
\hline Sodium & $\mathrm{Na}$ & 2310 & $71.613 .10^{-3}$ \\
\hline Magnesium & $\mathrm{Mg}$ & 2412 & $21.778 .10^{-4}$ \\
\hline \hline Aluminum & $\mathrm{Al}$ & 2711 & $16.147 .10^{-3}$ \\
\hline \hline Scandium & $\mathrm{Sc}$ & 4517 & $28.923 .10^{-3}$ \\
\hline Titanium & $\mathrm{Ti}$ & 4810 & $16.649 .10^{-3}$ \\
\hline \hline Vanadium & $\mathrm{V}$ & 5117 & $12.656 .10^{-3}$ \\
\hline \hline Chromium & $\mathrm{Cr}$ & 5224 & $35.013 .10^{-4}$ \\
\hline
\end{tabular}

\section{Conclusion}

The lateral profile of Cherenkov radiation in EAS initiated by several primaries such as (H, He, Li, Be, Ne, Na, Mg, Al, Sc, Ti, V and $\mathrm{Cr}$ ) has been simulated by CORSIKA program at the energy $3 \mathrm{PeV}$ for conditions of Tunka133 array. Basing on the simulated results, sets of parameterized functions were constructed by depending on Breit-Wigner model as a function of particle's type. The comparison between the approximated lateral distributions of Cherenkov radiation with that simulated using CORSIKA code for Tunka-133 array is verified for several primary particles. This comparison proved the ability of particle identification producing an extensive air showers and energy determination and mass composition around the knee region of the cosmic ray spectrum. This approach gives a significane of making a library of lateral distributions of Cherenkov radiation within a short time that could be utilized for real events analysis that have been detected in the experimental arrays and for energy spectrum and mass composition reconstructing for high energy cosmic rays.

\section{References}

[1] Freier, P. S. and Waddington, C. J. "The Cascading of Cosmic-Ray Nuclei in various media", Astrophysics and Space Science, 38,( 2), 419-436, 1975. 
[2] Khristiansen,G. B., Kulikov,G. V., and Fomin,Y. A. "Ultra-High-Energy Cosmic Radiation", Atomizdat, Moscow, 256, 1975.

[3] Jelly, J. V., "Cherenkov radiation and its Applications", London: Pergamon Press, 1958.

[4] Fomin Yu. A., and Khristiansen, G.B., "Study of the longitudinal development of individual EAS inferred from the Cherenkov light pulse shape: method and results, Nucl. Instrum". Methods Phys. Res. A., 248, 227-233, 1986.

[5] Prosin, V., Berezhnev, S.F., Budnev N.M.,et al., "Tunka-133: main experimental results of 3 year operation", The Astroparticle Physics conference, 33rd International Cosmic Ray Conference (ICRC), Rio De Janeiro, 2013.

[6] Rastegarzadeh, G., and Khoshabadi, S., "An investigation of using hump in the Cherenkov photon lateral distribution for Gamma-Hadron separation", Iranian Journal of Astronomy and Astrophysics, 1(2), 127-137, 2014.

[7] Mitri, I. De, "Measurement of the cosmic ray all-particle and light-component energy spectra with the ARGO-YBJ experiment", EPJ Web of Conferences 99, 08003, 2015.

[8] Budnev, N.M., Chernov, D. V., Gress, O. A. et al., "Cosmic ray energy spectrum and mass composition from $10^{15} \mathrm{eV}$ to $10^{17} \mathrm{eV}$ by data of the Tunka EAS Cherenkov array", 29th International Cosmic Ray Conference (ICRC), Pune,.6, 257-260, 2005.

[9] Al-Rubaiee, A.A., Hashim, U., Arshad, M.K Md et al., "Estimating of Cherenkov radiation in Extensive Air Showers using CORSIKA code for Tunka133 EAS Cherenkov array", Applied Mechanics and Materials, 754-755, 807-811, 2015.

[10] Alexandrov, L., Mavrodiev, S. Cht., Mishev, A. and Stamenov, J., "Estimation of the primary cosmic radiation characteristics", In Proc. 27th International Cosmic Ray Conference (ICRC), Hamburg, 1, 257-260, 2001.

[11] Mavrodiev, S. C., Mishev, A. L. and Stamenov, J. N., "A method for energy estimation and mass composition determination of primary cosmic rays at the Chacaltaya observation level based on the
Atmospheric Cherenkov Light technique", Nuclear Instruments and Methods in Physics Research A: Accelerators, Spectrometers, Detectors and Associated Equipment, 530(3), 359-366, 2004.

[12] Nerling F., Blumer J., Engel R., Risse M. "Description of Cherenkov light production in high-energy air showers", Astropart. Phys. ,24, 421-437, 2006.

[13] Angelov I., Duverger E., Makovicka L. et al. "Modeling and study of the Cerenkov effect//https://arxiv.org/abs/physics/031104 $4 \mathrm{v} 2,2003$.

[14] Nerling F., Blumer J., Engel R., Risse M. "Universality of electron distributions in high-energy air showers - Description of Cherenkov light production", Astropart. Phys., 24.,421-437, 2006.

[15] Heck D. and Peirog T., "Extensive air shower simulations at the highest energies", A user's Guide, Institut fur Kernphysik, Germany, 2013.

[16] Ostapchenko S., Nucl. Phys. B. Proc. Suppl., 151, 143-146, 2006.

[17] Heck D. and Engel R., In Proc. $28^{\text {th }}$ ICRC, Tsukuba, 279-282, 2003.

[18] Mishev A. et al., Nucl. Instrum. Meth., 474, 101-107, 2001.

[19] Al-Rubaiee, A.A., Gress, O.A. Kochanov, A.A., Lokhtin, K.S., Parfenov Y.V. and Sinegovsky, S.I, "Parameterization for Cherenkov light lateral distribution function in Extensive Air Showers", Proc. 29th International Cosmic Ray Conference, Pune, 249-252, 2005. 